\title{
Structural changes in bacteriorhodopsin induced by electric impulses
}

\author{
Kinko Tsuji and Eberhard Neumann \\ Max Planck Institut für Biochemie, D-8033 Martinsried/München, W. Germany
}

(Received 16 April 1980; revised 10 June 1980)

\begin{abstract}
Electric impulses of high field intensity $\left(2 \times 10^{5}\right.$ to $3 \times 10^{6} \mathrm{Vm}^{-1}, 1$ to $20 \mu \mathrm{s}$ duration $)$ cause transient changes in the optical absorbance of suspended purple membranes of Halobacterium halobium. The electric dichroism at 1 $m \mathrm{M} \mathrm{NaCl}, \mathrm{pH} \sim 6$ and at $293 \mathrm{~K}$ is dependent on field strength, pulse duration and wavelength of the monitoring, plane-polarized light in the range 400 to $650 \mathrm{~nm}$. The optically detected processes are, however, independent of bacteriorhodopsin concentration, of ionic strength and of the intensity of the monitoring light: These data together with the analysis of time course and steady state of the reduced dichroism, suggest electric field-sensitive, intramembraneous structural changes which lead to restricted orientation changes of the chromophore. $A$ theoretical analysis of restricted orientation is developed and applied to the electro-optic data. As a result, it is found that the electric dichroism of purple membranes is associated with a large polarizability anisotropy of 2.4 $\times 10^{-30} \mathrm{Fm}^{2}\left(2.2 \times 10^{-14} \mathrm{~cm}^{3}\right)$; the electric permanent dipole moment which is involved amounts to $4.7 \times 10^{-28}$ C m(140 Debye). The kinetic data suggest a cyclic reaction scheme with at least five different conformations. The high polarizability is probably due to displaceable ionic groups within the cooperative lattice of bacteriorhodopsin molecules in purple membranes.
\end{abstract}

\section{Introduction}

Bacteriorhodopsin is the photoactive protein in the purple membrane, which is a specialized part of the cell membrane of many halobacteria ${ }^{1}$. The protein functions as a light-driven proton pump producing an electrochemical proton gradient from which the free energy of the light induced ATP synthesis is finally derived ${ }^{2}$. As an integral membrane protein, bacteriorhodopsin is exposed to the internal electric field of the membrane. Isolated purple membrane fragments may be subjected to external electric impulses in order to study the structural and functional effects both of the intrinsic electric field and of variations in the membrane field. Provided that structural changes induced by the electric field are accompanied by variations in the chromophore and its protein environment, specific conformational transitions should be optically measurable. Light transmission changes have been observed when field pulses are applied to purple membranes ${ }^{3-6}$ as well as to apomembrane fragments ${ }^{4}$. However, the type and mechanism of the optical signal changes have not been analysed.

If the applied electric fields last longer ${ }^{3,5,7}$, orientation of whole membrane fragments will contribute to the optical signal. Using rectangular electric pulses of short duration, the contribution of fragment orientation is negligible and does not interfere with electro-optical signals arising from intramembraneous processes. In this paper we report on electric field-induced absorbance changes, which reflect both structural transitions and rotational changes in the chromophore within purple membranes. The electro-optical signals suggest that the orientation changes in the chromophore are sterically restricted and are accompanied by a change in the optical extinction coefficient, resulting in a chemical contribution to the absorbance change. For the data analysis an electro-optic theory for restricted orientation is developed. The main finding of this study is that external electric fields induce intramolecular structural changes in purple membranes and these lead to restricted rotation of the chromophore coupled to a very large induced dipole as well as to an electric permanent dipole moment. The order of magnitude of the electric moments at the field strengths applied indicate cooperative transitions of bacteriorhodopsin in purple membranes. An electric impulse causes a cyclic change through at least five different conformations of the protein.

\section{Theory}

The experimental data indicate that bacteriorhodopsin in purple membranes is electro-optically anisotropic. The chromophore is a part of a rather rigid protein-lipid structure $^{8}$, hence orientational displacements of the chromophore within the protein are expected to be sterically restricted. Limited orientation differs from the rotations of freely mobile particles and requires appropriate theoretical analysis.

It is known that the angle, $\psi$, between the chromophore transition moment $\mu$ of bacteriorhodopsin and the membrane normal is $\sim 1.2 \mathrm{rad}\left(70^{\circ}\right)^{9-11}$. The electro-optic data of the present study suggest that an electric field $E$ causes an angular displacement $\Delta \psi$ of $\mu$ from the position $\psi^{0}$ in the absence of a field. As shown in Figure la, as a result of steric restriction, for instance, $\Delta \psi$ may be limited to a maximum value, $\Delta \psi_{m}$. Generally, two limiting cases of orientational restriction may be considered for the membrane-bound proteins, when the membrane fragments themselves remain randomly distributed. 

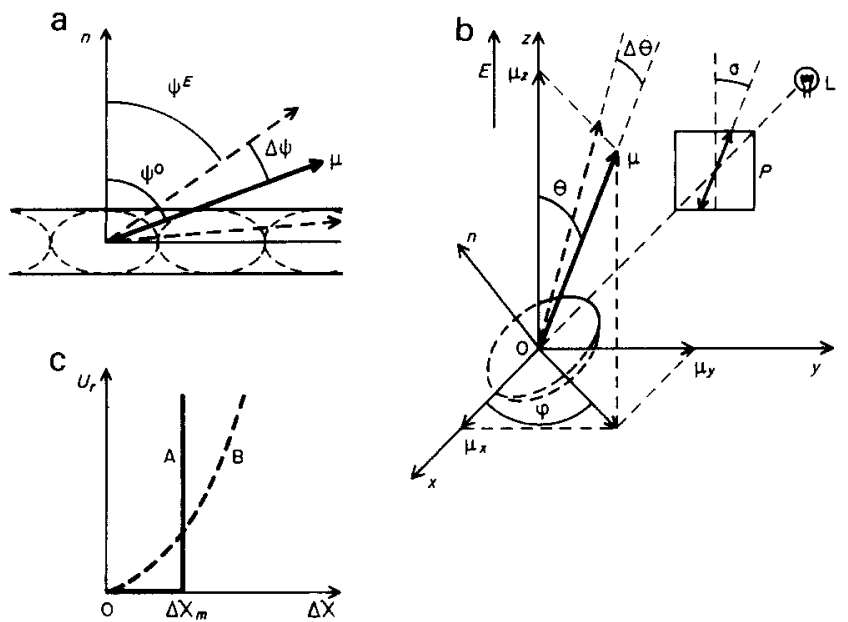

Figure 1 (a) Cross-section of purple membrane; parallel to the membrane normal $n ; \mu$, optical transition moment; $\Delta \psi$ is the angular displacement in an electric field $E$. Case I: $\mu$ can rotate symmetrically toward and away from $n$ such that $\psi^{E}=\psi^{0} \pm \Delta \psi$. Case II: $\mu$ can rotate to only one side, e.g. $\psi^{E}=\psi^{0}-\Delta \psi$ leading to positive dichroism. (b) Geometrical relations of electric dichroism in Cartesian coordinates. $E$ is in the $z$-direction, the light beam from a source $L$ is in the $x$-axis and the polarizer, $P$, is in the $y, z$-plane providing plane-polarized light under the angle $\sigma$ relative to $E$. The optical transition moment $\mu$ of a particular chromophore lies under the angle $\theta$ to $E$. (c) Dependence of the restriction energy term $U_{r}$ on the angular displacement $\Delta \chi$ for model $A$ (well-type potential energy profile) and model B (potential energy according to Hooke's law); see equations (12) and (13) of the text

Case I: $\mu$ may be displaced in both directions relative to the membrane normal, either increasing or decreasing $\psi$. Hence, this angular displacement is symmetric and, in the field, we obtain $\psi^{E}=\psi^{0} \pm \Delta \psi$.

Case II: $\mu$ can rotate in one direction only. This asymmetric displacement leads to $\psi^{E}=\psi^{0}-\Delta \psi$ for positive dichroism and, alternatively, to $\psi^{E}=\psi^{0}+\Delta \psi$ for negative dichroism. If case II applies, only one half of the membrane fragments are affected by the electric field.

\section{Orientation factor of restricted rotation}

The formula to calculate the orientation factor for restricted orientation is analogous to that for free, unrestricted orientation. Therefore, the key equations for free orientation $^{12}$ are given first.

The absorbance change, $\Delta A_{\sigma}$, caused by the electric field, $E$, in an electro-optically anisotropic system, measured with light that is plane-polarized under the angle $\sigma$ with respect to $E$ (see Figure $1 b$ ), is given by:

$$
\Delta A_{\sigma}=A_{\sigma}^{E}-A
$$

where $A_{\sigma}^{E}$ is the absorbance in the presence of the field and $A$ is the absorbance at $E=0$.

The reduced absorbance change is generally expressed in terms of the orientation factor, $\Phi$, according to:

$$
\frac{\Delta A_{\sigma}}{A}=\frac{1}{2}\left(3 \cos ^{2} \omega-1\right)\left(3 \cos ^{2} \sigma-1\right) \Phi
$$

where $\omega$ is the fixed angle between optical transition moinent $\mu$ and the electrical symmetry axis of the orienting unit (permanent and induced dipoles) ${ }^{13}$.

The reduced dichroism is defined by:

$$
\frac{\Delta A}{A}=\frac{A_{\|}^{E}-A_{1}^{E}}{A}=32\left(3 \cos ^{2} \omega-1\right) \Phi
$$

where $A_{\|}^{E}$ and $A_{\perp}^{E}$ are the absorbance values measured in the parallel $(\sigma=0)$ and in the perpendicular $(\sigma=\pi / 2)$ modes of light polarization, respectively.

The orientation factor is given by:

$$
\Phi=\frac{1}{2}\left(3<\cos ^{2} \chi>-1\right)
$$

where $\chi$ is the angle between the electric symmetry axis of the orienting unit and the direction of the external electric field. The average value of $\cos ^{2} \chi$ is defined by:

$$
\left\langle\cos ^{2} \chi\right\rangle=\int_{0}^{\pi} \cos ^{2} \chi f_{\chi} 2 \pi \sin \chi \cdot \mathrm{d} \chi
$$

and the angular distribution function of the orienting unit for the steady state is given by:

$$
f_{\chi}=\frac{\exp (-U / k T)}{\int_{0}^{\pi} \exp (-U / k T) 2 \pi \sin \chi \cdot \mathrm{d} \chi}
$$

where $k$ is the Boltzmann constant and $T$ is the absolute temperature.

The potential energy, $U$, of a freely orienting unit characterized by the permanent dipole moment $p$ and by the polarizabilities $\alpha_{1}$ (parallel to the axis of electric symmetry) and $\alpha_{2}$ (perpendicular to it) is given by:

$$
U=-p E_{d} \cos \chi-\frac{1}{2}\left(\alpha_{1}-\alpha_{2}\right) E_{i}^{2} \cos ^{2} \chi
$$

where, to a first approximation, we assume that the directing field $E_{d}$ and the internal field $E_{i}$ are equal to the external field $E$ (see Discussion). Introducing equations (5) to (7) into equation (4) yields the familiar relationship:

$$
\Phi=\frac{3 \int_{-1}^{1} u^{2} \exp \left(\beta u+\gamma u^{2}\right) \mathrm{d} u}{2 \int_{-1}^{1} \exp \left(\beta u+\gamma u^{2}\right) \mathrm{d} u}-\frac{1}{2}
$$

where:

$$
\begin{aligned}
& u=\cos \chi \\
& \beta=p E_{d} / k T \\
& \gamma=\left(\alpha_{1}-\alpha_{2}\right) E_{i}^{2} /(2 k T)
\end{aligned}
$$

For restricted orientation, the potential energy may be written in two terms: 


$$
U=U_{0}+U_{r}
$$

where $U_{0}$ has the form of equation (7) and the restriction term $U_{r}$ specifies the type of steric restriction. Clearly, for unrestricted, free orientation, $U_{r}=0$ and $U=U_{0}$. In general, with respect to $\chi$, orientation is viewed as an angular displacement $\Delta \chi$ of the axis of electric symmetry of the orienting unit from a position $\chi^{0}$ at $E=0$ to a position $\chi^{E}$ in the presence of the field; hence $\Delta \chi=\chi^{0}-\chi^{E}$ $\geqslant 0$. Whereas in unrestricted, free orientation, $\Delta \chi$ does not enter into the energy term, restricted orientation may be explicitly expressed as a function of $\Delta \chi$. Further analysis in this study will be confined to the theoretical treatment of two physically simple models.

Model A: the steric restriction is approximated by a well-type energy profile for $U_{r}$ with infinitely high boundaries. The angular displacement is therefore subjected to the condition $0 \leqslant \Delta \chi \leqslant \Delta \chi_{m}$, where $\Delta \chi_{m}$ is the maximum displacement which corresponds to a maximum $\Delta \psi_{m}$ for the displacement of the chromophore $\mu$ relative to the membrane normal; see Figure la. The restriction term $U_{r}$ is then

$$
U_{r}=0 \text { for } 0 \leqslant \Delta \chi \leqslant \Delta \chi_{m}, \text { and } U_{r}=\infty \text { for } \Delta \chi>\Delta \chi_{m}
$$

Model $B$ : the angular motion of the orienting unit is hindered and is viewed as a torque, $M_{c}$, which is opposite to the orienting torque exerted by the external electric field. In the simplest, case, this counter-torque may be expressed in terms of Hooke's law, according to $M_{c}=a \cdot \Delta \chi$, where $a$ is a constant and, therefore, the restriction term is given by:

$$
U_{r}=\frac{1}{2} a(\Delta \chi)^{2}
$$

The energy profiles of $U_{r}$ for these two models are shown in Figure 1c.

The total potential energy terms for the two models are summarized as follows:

Model A:

$$
\begin{gathered}
U=-p E \cos \chi^{E}-\frac{1}{2}\left(\alpha_{1}-\alpha_{2}\right) E^{2} \cos ^{2} \chi^{E} \\
\text { for }\left(\chi^{0}-\Delta \chi_{m}\right) \leqslant \chi^{E} \leqslant\left(\chi^{0}+\Delta \chi_{m}\right) \\
U=\infty \text { for } 0 \leqslant \chi^{E}<\left(\chi^{0}-\Delta \chi_{m}\right) \text { or } \chi^{E}>\left(\chi^{0}+\Delta \chi_{m}\right)
\end{gathered}
$$

\section{Model B}

$$
U=-p E \cos \chi^{E}-\frac{1}{2}\left(\alpha_{1}-\alpha_{2}\right) E^{2} \cos ^{2} \chi^{E}+\frac{1}{2} a\left(\chi^{0}-\chi^{E}\right)^{2}
$$

These potential energies are a function of two variables, $\chi^{0}\left(0 \leqslant \chi^{0} \leqslant \pi\right)$ and $\chi^{E}\left(0 \leqslant \chi^{E} \leqslant \chi^{0}\right)$; note that $\chi^{0}$ is independent of $\chi^{E}$. Therefore, we may introduce a partial orientation factor $\Phi_{\chi^{0}}$ for the particular angular position $\chi^{0}$. Then, the orientation factor $\Phi$ can be obtained as the average of $\Phi_{x^{0}}$ for all angular directions $\chi^{0}$ :

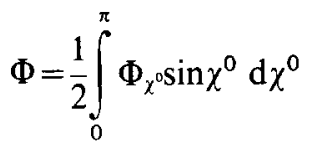

Note that the orientation factor is reduced by a factor of $1 / 2$ in case II, because for $\pi / 2<\chi^{0} \leqslant \pi, \Phi_{\chi^{0}}=0$, i.e. for one-half of the system there is no orientation.

The partial orientation factor is now obtained from equations (4) to (9) with $\chi=\chi^{E}$. For the models A and B, equations (12) and (13) are introduced into equation (6), respectively. The respective partial orientation factors are for:

Model A

$$
\Phi_{x^{0}}=\frac{3 \int_{u_{0}}^{u_{m}} u^{2} \exp \left(\beta u+\gamma u^{2}\right) \mathrm{d} u}{2 \int_{u_{0}}^{u_{m}} \exp \left(\beta u+\gamma u^{2}\right) \mathrm{d} u}-\frac{1}{2}
$$

with $u_{0}=\cos \left(\chi^{0}+\Delta \chi_{m}\right)$ and $u_{m}=\cos \left(\chi^{0}-\Delta \chi_{m}\right)$ and for:

Model B:

$$
\Phi_{\chi^{0}}=\frac{3 \int_{0}^{\pi} u^{2} \exp \left\{\beta u+\gamma u^{2}-g(u)\right\} \mathrm{d} u}{2 \int_{0}^{\pi} \exp \left\{\beta u+\gamma u^{2}-g(u)\right\} \mathrm{d} u}-\frac{1}{2}
$$

$$
\begin{aligned}
g(u) & \simeq a(1-\cos \Delta \chi) / k T \\
& =a\left(1-u \cos \chi^{0}-\left(1-u^{2}\right)^{1 / 2} \sin \chi^{0}\right) / k T
\end{aligned}
$$

The results of numerical calculations of equation (14) for the symmetric case I and the asymmetric case II are shown in Figure 2, using model A (well-type energy profile) with $\Delta \chi_{m}$ as a parameter and model $\mathrm{B}$ (Hooke-type energy profile) with $a / k T$ as a parameter, respectively. Note that the variable $\left(\beta^{2}+2 \gamma\right)$ with the permanent $(\beta)$ and the induced $(\gamma)$ dipole terms defined by equations $(9 \mathrm{~b})$ and $(9 \mathrm{c})$, is proportional to $E^{2}$. Therefore the field strength dependence of the reduced dichroism can be curve-fitted to the function $\Phi\left(\beta^{2}+2 \gamma\right)$. Hence, the mechanism as well as the type of orientation (free or restricted) may be determined.

\section{Electric dichroism of restricted orientation}

The primary data of electric dichroism of systems like purple membranes are related to the angle $\theta$ between the optical transition moment, $\mu$, of a particular chromophore (or clusters of chromophores) and the direction of the external electric field, see Figure $1 b$. For simplicity we shall at first assume that the electric symmetry axis is parallel to the optical transition moment $(\omega=0)$. This assumption is in line with the observation of positive dichroism; see equation (3). Furthermore, as demo- 

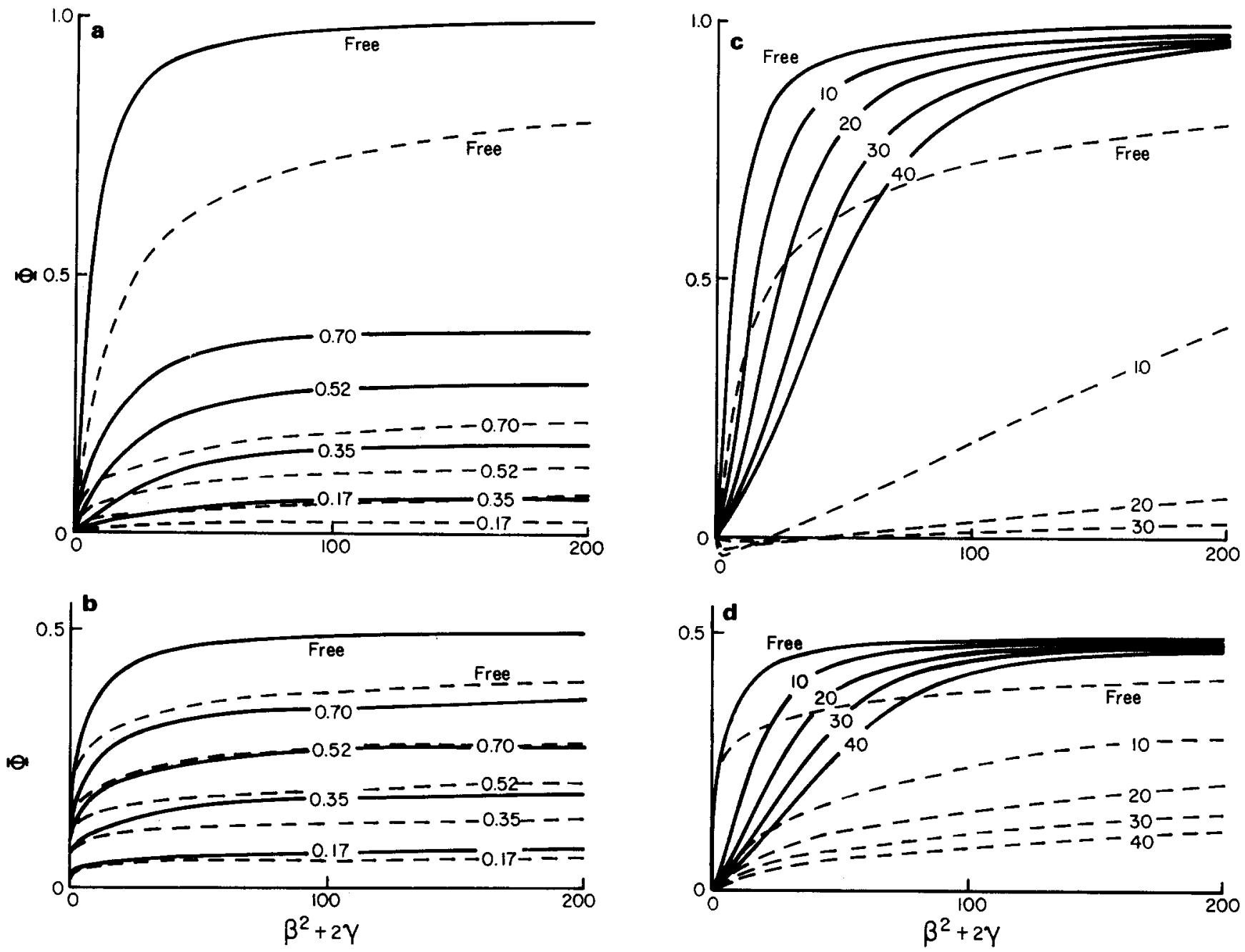

Figure 2 Orientation factor $\Phi$ for restricted angular displacements $\Delta \chi_{m}$ of the electric axis as a function of $\beta^{2}+2 \gamma$; for comparison, unrestricted free orientation is included. ---, Refers to $\Phi$ due to pure permanent dipoles, i.e. $\gamma=0$; --, refers to $\Phi$ due to induced dipoles, i.e. $\beta=0, \gamma>0$. (a) and (b) refer to cases I and II, respectively, assuming the restriction energy model A for various maximum angular displacements $\Delta \chi_{m}$ as a parameter. (c) and (d) refer to cases I and II, respectively, assuming the restriction energy model B for various values of $a / k T$

nstrated below, the experimental data on membranebound bacteriorhodopsin can be consistently analysed in terms of an axially symmetric overall electric moment. For $\omega=0$, the boundary conditions for $\theta$ are the same as those for $\chi$ discussed in the previous section.

As visualized in Figure $1 b$ the Cartesian components of $\mu$ are given by:

$$
\begin{aligned}
& \mu_{x}=\mu \sin \theta \cos \varphi \\
& \mu_{y}=\mu \sin \theta \sin \varphi \\
& \mu_{z}=\mu \cos \theta
\end{aligned}
$$

In the absence of an external electric field the components of $\mu$ and the angle $\theta$ are denoted by $\mu_{x}^{0}, \mu_{y}^{0}, \mu_{z}^{0}$, and $\theta^{0}$, whereas in the applied field the superscript zero is replaced by the superscript $E$.

The optical absorbance $A_{\sigma}$ for light that is planepolarized under the angle $\sigma$ to the direction of the electric field (see Figure $l b$ ) is, for a small anisotropy, given by:

$$
A_{\sigma}=\frac{\kappa}{4 \pi} \int_{0}^{\pi} \int_{0}^{2 \pi}\left(\mu_{y} \sin \sigma+\mu_{z} \cos \sigma\right)^{2} \mathrm{~d} \varphi \sin \theta^{0} \mathrm{~d} \theta^{0}
$$

where $\kappa$ is a constant ${ }^{13}$

In the absence of an electric field, where the chromophores can be considered as randomly distributed, the absorbance is independent of $\sigma$; equation (18) is reduced to:

$$
A_{\sigma}^{0}=A=\frac{1}{3} \kappa \mu^{2}
$$

In the presence of a field, electro-optical anisotropy is indicated by an absorbance value that characteristically depends on $\sigma$ and on $E$.

For the two limit cases of restricted angular displacements, equation (18) is rewritten in terms of $\mu_{y}^{E}$ and $\mu_{z}^{E}$, according to equations (17). Then, $\theta^{E}$ is expressed in terms of $\theta^{0}$ in the same way as for $\chi$.

For model A, further treatment is particularly simple if the saturation value $\Phi_{s}$ of the orientation factor is considered. At sufficiently high external electric fields, all chromophore transition moments which have orientations between $\theta^{\circ}=0$ and $\theta^{\circ}=\Delta \theta_{m}$ are parallel to $E$, i.e. $\theta^{E}=0$. The remaining molecules whose transition moments are positioned between $\theta^{\circ}=\Delta \theta_{m}$ and $\theta^{\circ}=\pi$ assume the positions $\theta^{E}=\theta^{0}-\Delta \theta_{m}$ for the permanent dipole, while $\theta^{E}=\theta^{0}-\Delta \theta_{m}$ when $\theta^{0}$ is between $\Delta \theta_{m}$ and $\pi / 2$ and 
$\theta^{E}=\theta^{0}+\Delta \theta_{m}$ when $\theta^{0}$ is between $\pi / 2$ and $\pi=\Delta \theta_{m}$ for the induced dipole.

The relations between $\theta^{0}$ and $\theta^{E}$ for Case $I$ are for permanent dipoles:

$$
\begin{array}{ll}
\theta^{E}=0 & \text { for } 0 \leqslant \theta^{0} \leqslant \Delta \theta_{m} \\
\theta^{E}=\theta^{0}-\Delta \theta_{m} & \text { for } \Delta \theta_{m}<\theta^{0} \leqslant \pi
\end{array}
$$

and for induced dipoles:

$$
\begin{array}{ll}
\theta^{E}=0 & \text { for } 0 \leqslant \theta^{0} \leqslant \Delta \theta_{m} \text { or } \pi-\Delta \theta_{m} \leqslant \theta^{0} \leqslant \pi \\
\theta^{E}=\theta^{0}-\Delta \theta_{m} & \text { for } \Delta \theta_{m}<\theta^{0}<\pi / 2 \\
\theta^{E}=\theta^{0}+\Delta \theta_{m} & \text { for } \pi / 2<\theta^{0} \leqslant\left(\pi-\Delta \theta_{m}\right)
\end{array}
$$

Applying equations (20a) and (20b) to $\mu_{y}$ and $\mu_{z}$, in equation (17) we obtain the absorbances in the saturation region (subscript $s$ ) for permanent dipoles:

$$
\begin{aligned}
&\left(A_{\sigma}^{E}\right)_{s}=\frac{\kappa}{4 \pi} \int_{0}^{\pi} \int_{0}^{2 \pi}\left(\mu_{y}^{E} \sin \sigma+\mu_{z}^{E} \cos \sigma\right)^{2} \mathrm{~d} \varphi \sin \theta^{0} \mathrm{~d} \theta^{0} \\
&= \frac{\kappa \mu^{2}}{2}\left[\left\{\int_{0}^{\Delta \theta_{m}} \sin \theta^{0} \mathrm{~d} \theta^{0}+\int_{\Delta \theta_{m}}^{\pi} \cos ^{2}\left(\theta^{0}-\Delta \theta_{m}\right) \sin \theta^{0} \mathrm{~d} \theta^{0}\right\} \cos ^{2} \sigma\right. \\
&\left.+\frac{1}{2}\left\{\int_{\Delta \theta_{m}} \sin ^{2}\left(\theta^{0}-\Delta \theta_{m}\right) \sin \theta^{0} \mathrm{~d} \theta^{0}\right\} \sin ^{2} \sigma\right] \\
&=\frac{\kappa \mu^{2}}{12}\left\{3\left(3-2 \cos \Delta \theta_{m}-\cos ^{2} \Delta \theta_{m}\right) \cos ^{2} \sigma+\right. \\
&\left.\left(1+2 \cos \Delta \theta_{m}+\cos ^{2} \Delta \theta_{m}\right)\right\}
\end{aligned}
$$

The corresponding expressions for induced dipoles are:

$$
\begin{gathered}
\left(A_{\sigma}^{E}\right)_{s}=\frac{\kappa \mu^{2}}{2}\left[\iint_{0}^{\Delta \theta_{m}} \sin \theta^{0} \mathrm{~d} \theta^{0}+\int_{\Delta \theta_{m}}^{\pi / 2} \cos ^{2}\left(\theta^{0}-\Delta \theta_{m}\right) \sin \theta^{0} \mathrm{~d} \theta^{0}\right. \\
\left.+\int_{\pi / 2}^{\pi-\Delta \theta_{m}} \cos ^{2}\left(\theta^{0}+\Delta \theta_{m}\right) \sin \theta^{0} \mathrm{~d} \theta^{0}+\int_{\pi-\Delta \theta_{m}}^{\pi} \sin \theta^{0} \mathrm{~d} \theta^{0}\right\} \cos ^{2} \sigma \\
+\frac{1}{2}\left\{\int_{\Delta \theta_{m}}^{\pi / 2} \sin ^{2}\left(\theta^{0}-\Delta \theta_{m}\right) \sin \theta^{0} \mathrm{~d} \theta^{0}\right. \\
\left.\left.+\int_{\pi / 2}^{\pi-\Delta \theta_{m}} \sin ^{2}\left(\theta^{0}+\Delta \theta_{m}\right) \sin \theta^{0} \mathrm{~d} \theta^{0}\right\} \sin ^{2} \sigma\right] \\
=\frac{\kappa \mu^{2}}{3}\left\{3\left(1-\cos \Delta \theta_{m}+\sin \Delta \theta_{m} \cos \Delta \theta_{m}\right) \cos ^{2} \sigma\right. \\
\left.+\left(1+\cos \Delta \theta_{m}-\sin \Delta \theta_{m} \cos \Delta \theta_{m}\right)\right\}
\end{gathered}
$$

The reduced absorbance changes and the reduced electric dichroism for maximum angular displacement of the chromophore are obtained by inserting equations (21a) or (21b) into equations (2) and (3), respectively. The reduced absorbance changes are, for permanent dipoles:

$$
\left(\frac{\Delta A_{\sigma}}{A}\right)_{s}=\frac{1}{4}\left(3-2 \cos \Delta \theta_{m}-\cos ^{2} \Delta \theta_{m}\right)\left(3 \cos ^{2} \sigma-1\right)(22 \mathrm{a})
$$

and for induced dipoles:

$$
\left(\frac{\Delta A_{\sigma}}{A}\right)_{s}=\left(1-\cos \Delta \theta_{m}+\sin \Delta \theta_{m} \cos \Delta \theta_{m}\right)\left(3 \cos ^{2} \sigma-1\right)
$$

The reduced dichroism for any values of $\omega$ are, for permanent dipoles:

$$
\left(\frac{\Delta A}{A}\right)_{s}=\frac{3}{8}\left(3 \cos ^{2} \omega-1\right)\left(3-2 \cos \Delta \theta_{m}-\cos ^{2} \Delta \theta_{m}\right)(23 a)
$$

and for induced dipoles

$$
\left(\frac{\Delta A}{A}\right)_{s}=\frac{3}{2}\left(3 \cos ^{2} \omega-1\right)\left(1-\cos \Delta \theta_{m}+\sin \Delta \theta_{m} \cos \Delta \theta_{m}\right)
$$

Provided that $\omega=0$, i.e. the optical and electrical axes are parallel, equation (23a) or (23b) permit the determination of $\Delta \theta_{m}$. Since for this case $\Delta \theta_{m}=\Delta \chi_{m}=\Delta \psi_{m}$, the maximum angular displacement relative to the membrane normal is obtained.

For model $\mathrm{B}$, the saturation value of the orientation factor is the same as for the free orientation. Therefore, the value of $\omega$ can be estimated from the experimental value of $(\Delta A / A)_{s}$ by using equation (3). However, the parameter $a$ can only be determined from the field strength dependence of $\Delta A / A$.

\section{Experimental}

\section{Materials}

Purple membranes isolated from the M1 strain of Halobacterium holobium were a gift from Professor Rosenheck, Weizmann Institute, Israel. Bacteriorhodopsin concentration in the purple membrane suspensions was determined on the basis of the molar absorption coefficient $\varepsilon=63000$ litre $\mathrm{mol}^{-1} \mathrm{~cm}^{-1}$ at $570 \mathrm{~nm}(\operatorname{Ref} 14)$.

The absorbance of purple membrane suspensions for the electric pulse measurements was adjusted to $\sim 0.3$ in $1 \mathrm{mM} \mathrm{NaCl}$ solution at $293 \mathrm{~K}$, unbuffered $(\mathrm{pH} \simeq 6$ ), unless otherwise stated. The absorbance value of 0.3 corresponds to a concentration which yields sufficiently large signals without serious light scattering contributions by the membrane fragments.

Measurements of electric field-induced absorbance changes

The electro-optic measurements were performed with an electric field-jump apparatus recently developed by Schallreuter and Neumann ${ }^{15}$.

The light source for the visible spectral range is a $100 \mathrm{~W}$ halogen lamp from which a collimated light beam passes through a Schoeffel-monochromator and a Nicoll polarizer that can be rotated from $\sigma=0$ to $\sigma=2 \pi$ with respect to the electric field. The sample cell (volume $\simeq 0.8 \mathrm{~cm}^{3}$ ) is similar to that used previously ${ }^{16}$. The distance between the flat electrodes is $13 \mathrm{~mm}$ and the path length of light is $7 \mathrm{~mm}$. The light intensity transmitted by the sample is detected by a photomultiplier and amplified. The output 


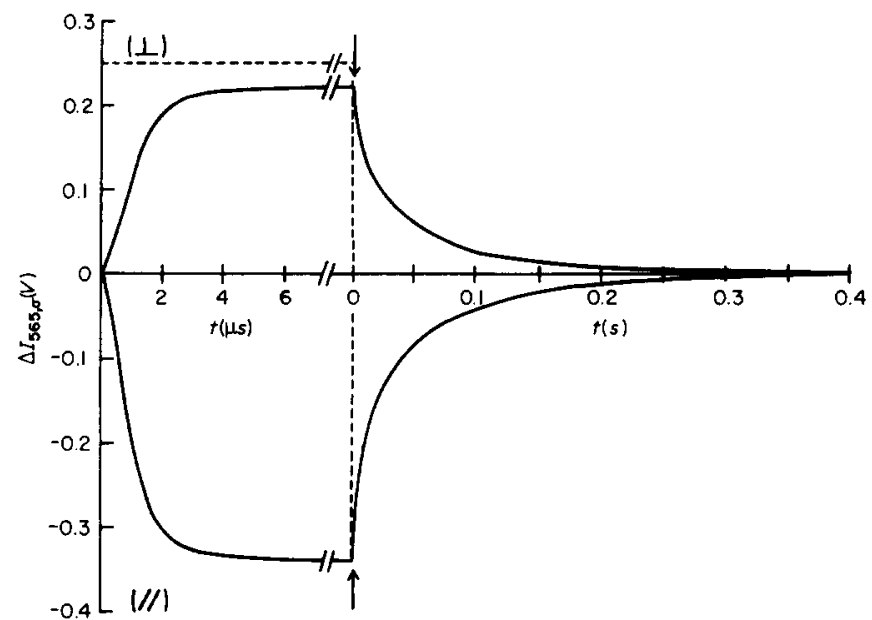

Figure 3 Electric dichroism of bacteriorhodopsin in purple membrane; concentration of bacteriorhodopsin $c=4.8 \times 10^{-6} \mathrm{M}$ in $1 \mathrm{mM} \mathrm{NaCl}$, unbuffered, $\mathrm{pH} \simeq 6, T=293 \mathrm{~K}$. The light transmittance change $\Delta I_{\sigma}$ at $\lambda=565 \mathrm{~nm}$ is caused by a rectangular electric pulse of $E=15.4 \times 10^{5} \mathrm{~V} \mathrm{~m}^{-1}$ applied at $t=0$ for $20 \mu$ s (see arrows). ( ||$)$ and $(1)$ are the signal changes for light plane-polarized parallel $(\sigma=0)$ and perpendicular $(\sigma=\pi / 2)$ to the electric field; the light intensity $I$ at $t=0$ is $3.0 \mathrm{~V}$

of the amplifier is displayed on an oscilloscope (Tekronix 7834 storage oscilloscope) and is simultaneously stored in a transient recorder (Datalab DL 922) which is connected to a computer (Interdata, modified by WDV, München).

Rectangular electric pulses up to $35 \mathrm{kV}$ and of various duration in the range 1 to $100 \mu \mathrm{s}$ were used. All measurements were carried out at $293 \mathrm{~K}$. The temperature increase due to Joule heating can be calculated according to:

$$
\Delta T=\frac{V^{2} \Delta t}{R v C_{p} \rho}
$$

where $R \simeq 1 \times 10^{4} \Omega$ is the sample cell resistance, $C_{p} \simeq 4.2$ $\mathrm{J}^{-1} \mathrm{~K}^{-1}$ is the specific heat of the suspension, $v \simeq 0.8$ $\mathrm{cm}^{3}$ is the cell volume and $\rho \simeq 1 \mathrm{~g} \mathrm{~cm}^{-3}$ is the density of the suspension. For the highest voltage $V=35 \mathrm{kV}$ and the longest pulse duration of $\Delta t=100 \mu \mathrm{s}$, the temperature increase $\Delta T=3.5 \mathrm{~K}$ is negligible, because the absorbance change from 293 to $296.5 \mathrm{~K}$ is $<3 \%$ (Ref. 5 ).

The change in the absorbance, $\Delta A_{\sigma}$, defined by equation (1) is calculated from the light transmittance at a given wavelength and a fixed light polarization mode $\sigma$ :

$$
\Delta A_{\sigma}=-\log \left(1+\Delta I_{\sigma} / I\right)
$$

where $I$ is the transmitted light in the absence of the electric field (independent of $\sigma$ ). The field-induced transmittance change is given by $\Delta I=I_{\sigma}^{E}-I$ where $I_{\sigma}$ is the light transmitted in the presence of the electric field. It is noted that in this study $\Delta I_{\sigma}$ is generally not small compared with $I$. Therefore, the approximation $\Delta A_{\sigma}=-0.4343 \Delta I_{\sigma} / I$ cannot be applied.

A simple peeling-off method was used to describe the continuous relaxation spectrum of the absorbance change after switching off the electric field.

\section{Results}

Figure 3 shows typical signal changes induced by an electric impulse in purple membrane suspensions at two light polarization modes. At an electric field strength of $E$ $=15.4 \times 10^{5} \mathrm{~V} \mathrm{~m}^{-1}$ a time-independent, steady-state level of light transmittance in the field (subscript ss) is reached only if the pulse duration is equal to or larger than $6 \mu \mathrm{s}$. For the parallel $(\sigma=0)$ and perpendicular $(\sigma=\pi / 2)$ polarization modes of the monitoring light, the reduced absorbance changes calculated according to equation (2) are:

$$
\left(\Delta A_{||} / A\right)_{s s}=0.32 \pm 0.02 \text { and }\left(\Delta A_{1} / A\right)_{s s}=-0.27 \pm 0.02
$$

As seen in Figure 3, the signal change, after the electric field is switched off, reflects a continuous relaxation spectrum. At the present accuracy of data recording, the corresponding absorbance curves may be formally described in terms of at least four exponential components according to:

$$
\frac{\Delta A(t)}{\Delta A_{s s}}=\sum_{i=1}^{n} \delta_{i} \exp \left(-t / \tau_{i}\right) \quad(n \geqslant 4)
$$

where $\Delta A_{\text {ss }}$ is the steady-state absorbance change (when the pulse is terminated) and $\delta_{i}$ and $\tau_{i}$ are the relative amplitude and relaxation time of component $i$, respectively. For the curve in Figure 3 it is found that $\tau_{1}=0.3$ $\pm 0.03 \mathrm{~ms}, \tau_{2}=3.0 \pm 0.3 \mathrm{~ms}, \tau_{3}=26 \pm 4 \mathrm{~ms}, \tau_{4}=100 \pm 10$ $\mathrm{ms} ; \delta_{1} \simeq 0.2, \delta_{2} \simeq 0 . \overline{2}, \delta_{3} \simeq 0.3, \delta_{4} \simeq 0.3$.

Compared to the time range of the rise up curve in the presence of the electric field, the field-off response is slower by four orders of magnitude. These experimental results already indicate that the optical data cannot reflect simple, free orientation of the chromophore.

Figure 4 shows the field strength dependence of the reduced absorbance change (steady-state) at $\lambda=565 \mathrm{~nm}$ for three light polarization modes; $\sigma=0$, parallel; $\sigma=\pi / 2$, perpendicular; $\sigma=0.955 \mathrm{rad}\left(54.7^{\circ}\right)$. Note that the absorbance change $\Delta A_{||}$apparently saturates at $E>4 \times 10^{5}$ $\mathrm{V} \mathrm{m}{ }^{-1}$ while $-\Delta A_{\perp}$ still increases with increasing field strength.

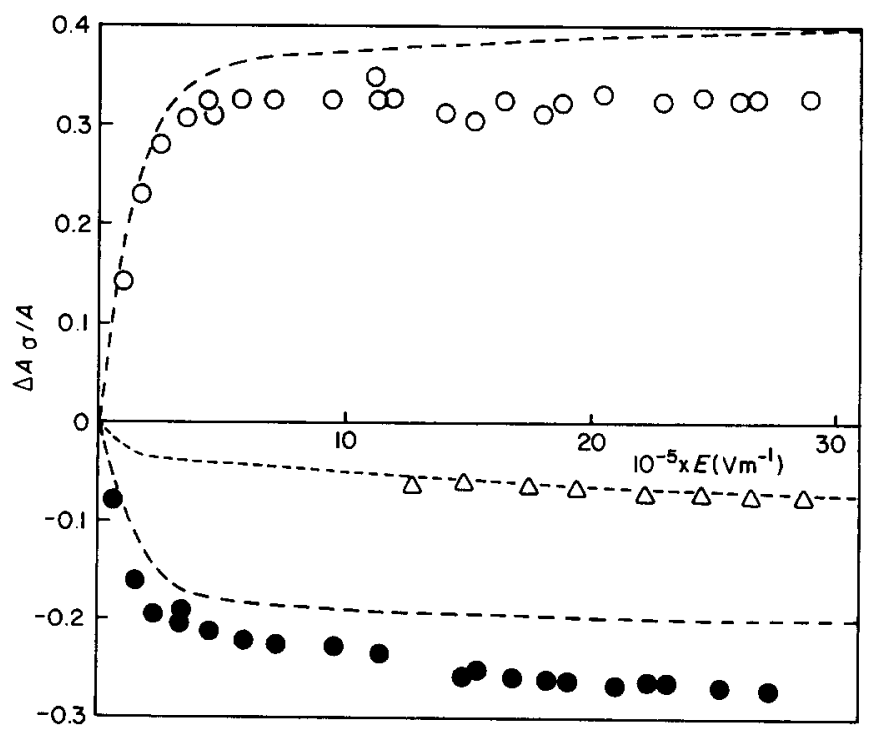

Figure 4 Electric field strength dependence of the steady-state value of the reduced absorbance change $\Delta A_{\sigma} / A$ for the purple membrane suspension at $\lambda=565 \mathrm{~nm}: \quad \circ, \sigma=0 ; \bullet, \sigma=\pi / 2$, and $\triangle, \sigma=0.955$. Broken lines were calculated according to equation (37): --- , refers to $\Delta A_{\sigma}^{(\text {rot })} / A$ at $\sigma=0$ and $\sigma=\pi / 2 ; \cdots$, refers to $\Delta A^{\text {(chem) }} / A$. Other experimental conditions are the same as in Figure 3 


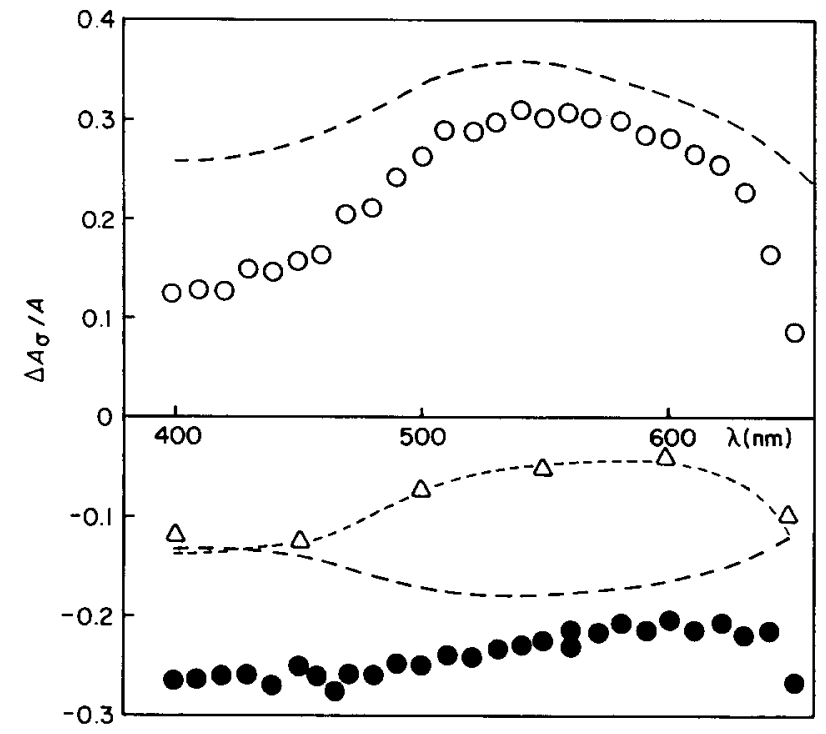

Figure 5 Wavelength dependence of the steady-state value of the reduced absorbance change $\Delta A_{\sigma} / A ; E=15.4 \times 10^{5} \mathrm{~V} \mathrm{~m}^{-1}$; $\circ$, at $\sigma=0 ; \bullet$, at $\sigma=\pi / 2 ; \Delta$, at $\sigma=0.955$. Broken lines were obtained according to equation (37):---, refers to $\Delta A_{\sigma}^{(\text {rot })} / A$ at $\sigma$ $=0$ and $\sigma=\pi / 2 ; \cdots$, refers to $\Delta A^{\text {(chem) } / A}$

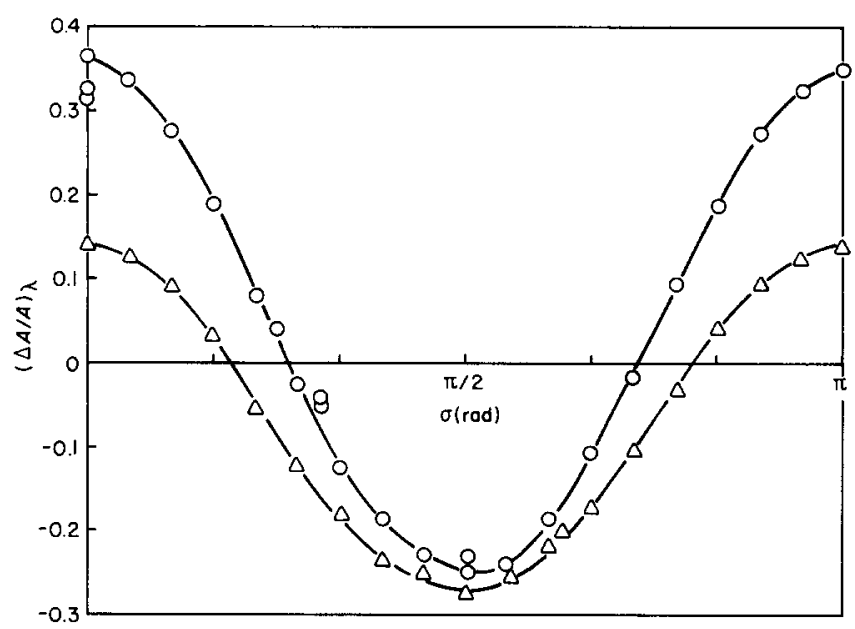

Figure 6 Dependence of the steady-state absorbance change of the purple membrane fragments $\Delta A_{\sigma} / A$ on the light polarization direction $\sigma$ with respect to the electric field at $\lambda=565 \mathrm{~nm}(0)$ and $400 \mathrm{~nm}(\triangle) ; E=15.4 \times 10^{5} \mathrm{~V} \mathrm{~m}^{-1}, 80 \mu$ s pulse duration

With freshly prepared purple membrane fragments, the signals are well reproducible; no damage is caused even by repetitive pulsing at $E=3 \times 10^{6} \mathrm{~V} \mathrm{~m}^{-1}$. With samples older than two months, repetitive pulses of $2 \times 10^{6} \mathrm{~V} \mathrm{~m}^{-1}$ caused an irreversible decrease in the absorbance change and a decrease in the relaxation times of the field-off response.

Figure 5 demonstrates that the reduced absorbance change (steady-state) depends on the wavelength of the monitoring light, in a different manner for the various polarization modes. In particular, the parallel mode has a pronounced maximum at $\sim 565 \mathrm{~nm}$. The ratio of the absorbance changes, $\left(\Delta A_{\mid} v A_{1}\right)$, is $-1.2 \pm 0.2$ at $565 \mathrm{~nm}$; at $400 \mathrm{~nm}$ this ratio is $-0.5 \pm 0.05$.

It is seen that $\Delta A_{0.955} / A$ is larger at $400 \mathrm{~nm}$ than at 565 nm. Figure 6 shows the reduced absorbance changes (steady-state) for two wavelengths, $565 \mathrm{~nm}$ and $400 \mathrm{~nm}$, as a function of the light polarization angle, $\sigma$. The apparent extinction angles are $\sigma_{565}=0.84 \mathrm{rad}\left(48^{\circ}\right)$ and $\sigma_{400}=0.61$ $\operatorname{rad}\left(35^{\circ}\right)$.

The reduced absorbance changes (steady-state) at $\sigma=0$ and $\sigma=\pi / 2$ are found to be independent of purple membrane concentration between $1.4 \times 10^{-6} \mathrm{M}$ and 2.0 $\times 10^{-5} \mathrm{M}$ and at ionic strengths between $1 \mathrm{mM}$ and $3 \mathrm{mM}$ $\mathrm{NaCl}$

As shown in Figure 7, the intensity of the monitoring light affects only very slightly the magnitude of the reduced absorbance changes. Thus, light-induced contributions to $\Delta A_{\sigma}$ are negligible.

Strikingly, the relaxation times of the field-off response are, within experimental error, independent of the field strength, pulse duration, wavelength, light polarization direction, concentration of purple membrane, ionic strength and the intensity of the monitoring light.

\section{Discussion}

Rotational and chemical contributions to the absorbance change

The absorbance per $\mathrm{cm}$ of a multicomponent system is generally given by the Beer-Lambert law:

$$
A=\sum_{i} \varepsilon_{i} c_{i}
$$

where $\varepsilon_{i}$ is the molar absorption coefficient and $c_{i}$ is the molar concentration of species $i$. It is recalled that the absorption coefficients of molecules which are intrinsically optically anisotropic, reflect average values $\bar{\varepsilon}_{i}$ of all chromophore orientations in the system.

When an electrical field is applied to a chemical system which exhibits both electrical and optical anisotropy, the

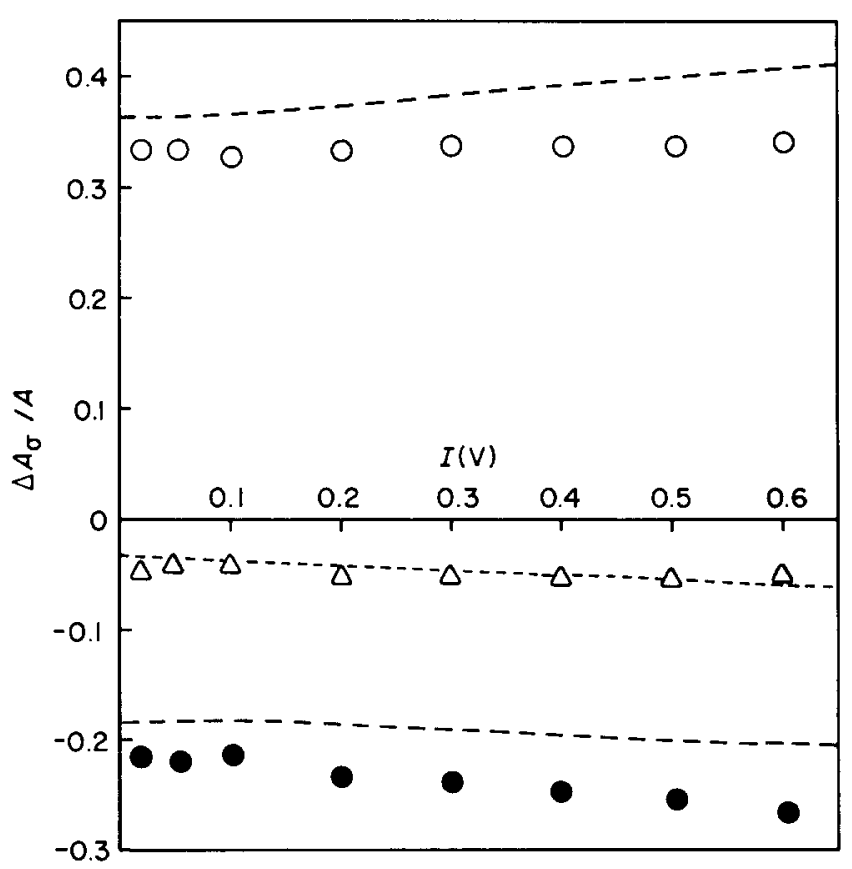

Figure 7 Dependence of the steady-state absorbance change of the purple membrane fragments $\Delta A_{\sigma} / A$ on the intensity of the monitoring light. $E=15.4 \times 10^{5} \mathrm{~V} \mathrm{~m}^{-1}, 80 \mu$ s pulse duration: 0 , $\sigma=0 ; \bullet, \sigma=\pi / 2 ; \Delta, \sigma=0.955$. Broken lines were calculated according to equation (37): --- , refers to $\Delta A_{\sigma}^{(\text {rot })} / A$ at $\sigma=0$ and $\sigma$ $=\pi / 2, \cdots$, refers to $\Delta A^{\text {(chem) }} / A$ 
field-induced absorbance change $\Delta A_{\sigma}$ may involve not only orientational changes $\left(\Delta \varepsilon_{i, \sigma}\right)$ but also concentration changes $\left(\Delta c_{i}\right)$.

In the absence of an electric field, the absorbance per $\mathrm{cm}$ of such a system is expressed by:

$$
A=\sum_{i} \bar{\varepsilon}_{i} c_{i}^{0}
$$

whereas $A$ is independent of $\sigma$, the absorbance in the field is $\sigma$-dependent:

$$
A_{\sigma}^{E}=\sum_{i} \varepsilon_{i, \sigma} \mathrm{c}_{i}^{E}
$$

Denoting the field-induced changes in $\varepsilon_{i}$ and $c_{i}$ by:

$$
\begin{aligned}
\Delta \varepsilon_{i, \sigma} & =\varepsilon_{i, \sigma}-\bar{\varepsilon}_{i} \\
\Delta c_{i} & =c_{i}^{E}-c_{i}^{0}
\end{aligned}
$$

equation (1) is rewritten in terms of equations (27) and (28):

$$
\begin{aligned}
\Delta A_{\sigma} & =\sum_{i}\left(\varepsilon_{i, \sigma} c_{i}^{E}-\bar{\varepsilon}_{i} c_{i}^{0}\right) \\
& =\sum_{i} \Delta \varepsilon_{i, \sigma}\left(c_{i}^{0}+\Delta c_{i}\right)+\sum_{i} \bar{\varepsilon}_{i} \Delta c_{i}
\end{aligned}
$$

where the separation of terms depending on $\sigma$ from those independent of $\sigma$ is apparent. Introducing the definitions:

$$
\Delta A_{\sigma}^{(\text {rot })}=\sum_{i} \Delta \varepsilon_{i, \sigma}\left(c_{i}^{0}+\Delta c_{i}\right)
$$

and:

$$
\Delta A^{(\text {chem })}=\sum_{i} \bar{\varepsilon}_{i} \Delta c_{i}
$$

equation (30) may be written generally in terms of a rotational and a chemical contribution:

$$
\Delta A_{\sigma}=\Delta A_{\sigma}^{(\text {rot })}+\Delta A^{(\text {chem })}
$$

For axially symmetric systems and small anisotropies, $\Delta A^{\text {(chem) }}$ can be experimentally obtained in two independent ways, using the absorbance changes $\Delta A_{\sigma}$ at the polarization modes $\sigma=0$ and $\sigma=\pi / 2$, or $\sigma=0.955 \mathrm{rad}$ $\left(54.7^{\circ}\right)$. In this case, according to equation (2), the relationship:

$$
\Delta A_{\|}^{(r o t)}+2 \Delta A_{\perp}^{(r o t)}=0
$$

holds, and for $\sigma=0.955$ rad equation (32) reduces to:

$$
\Delta A_{0.955}=\Delta A^{(\text {chem })}
$$

On the other hand, equations (32) and (33) can be combined to yield:

$$
\frac{1}{3}\left(\Delta A_{||}+2 \Delta A_{\perp}\right)=\Delta A^{(\text {chem })}
$$

Therefore, the equality:

$$
\Delta A_{0.955}=\frac{1}{3}\left(\Delta A_{||}+2 \Delta A_{\perp}\right)
$$

can be used as a criterion of axial symmetry of the electric dipole moment of the orienting unit.

Note that $\Delta A_{\sigma}^{(r o t)}$ specified in equation (31a) may contain concentration changes $\Delta c_{i}$. These changes $\left(\Sigma \Delta \varepsilon_{i, \sigma} \Delta c_{i}\right)$ are separable from the pure rotational term $\Sigma \Delta \varepsilon_{i, \sigma} c_{i}^{0}$, only when the kinetics of the rotational part are faster than the concentration changes ${ }^{17}$.

The primary experimental data, $\Delta A_{\sigma}$, on purple membranes do not follow equation (2), i.e. $\Delta A_{1}+2 \Delta A_{\perp} \neq 0$. Therefore, chemical changes may be involved. If, however, $\Delta A_{0.955}$ is compared with $\left(\Delta A_{\|}+2 \Delta A_{\perp}\right) / 3$, it is found that equation (36) holds. Hence, the system exhibits axial symmetry and equation (32) in the form:

$$
\Delta A_{\sigma}^{(r o t)}=\Delta A_{\sigma}-\Delta A_{0.955}
$$

can be used to calculate the rotational contributions of $\Delta A_{\sigma}$. The experimental data represented in Figures 4,5 and 7 are evaluated according to this subtraction procedure and the results are included as broken lines.

The wavelength dependence of $\Delta A_{\sigma}^{(r o t)} / A$ as represented in Figure 5 suggests that the absorption band between 400 and $650 \mathrm{~nm}$ arises from at least two different optical transitions. It is noted, too, that the chemical contribution $\left(\Delta A_{0.955} / A\right)$ is larger at $400 \mathrm{~nm}(-0.14 \pm 0.02)$ than at 565 $\mathrm{nm}(-0.055 \pm 0.005)$. It is seen in Figure 4 that the reduced changes in $\Delta A_{\|}^{(\text {rot })} / A$ and $-\Delta A_{\perp}^{(\text {rot })} / A$ increases with increasing field strength in qualitatively the same way as $\Delta A^{(\text {chem })} / A$.

The practical coincidence of $\Delta A_{\sigma}^{(\text {rot })}$ with $\Delta A^{(\mathrm{chem})}$ is reflected not only in the steady-state but also in the kinetics. As demonstrated in Figure 8, the entire timecourses of the relative signal changes in the various polarization modes, i.e. the rotational and the chemical contributions, are coincident within the margin of experimental error. These results suggest that $\Delta A^{(\text {chem })}$ and $\Delta A_{\sigma}^{(r o t)}$ reflect two aspects of one and the same process, which is induced by the electric field.

\section{Orienting unit}

As described above, the reduced absorbance changes are independent of bacteriorhodopsin concentration and of the ionic strength of the suspension. Therefore,

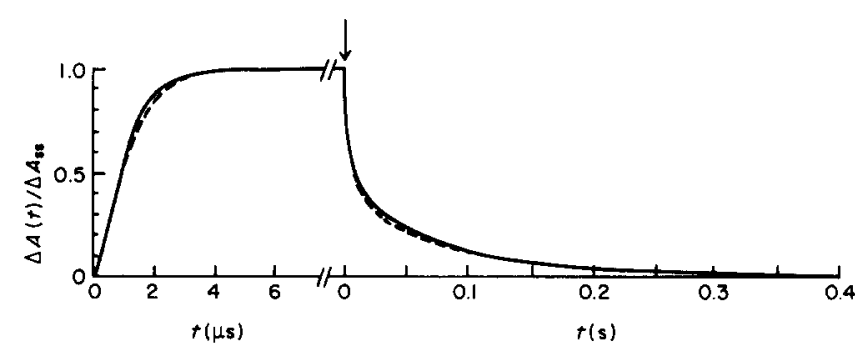

Figure 8 Time-course of the normalized electric dichroism $\Delta A^{(r o t)}(t) / \Delta A_{s s}^{(r o t)}=\left\{\Delta A_{||}(t)-\Delta A_{\perp}(t)\right\} / \Delta A_{s s}^{(r o t)}(\longrightarrow)$ and that of the normalized chemical contribution $\Delta A^{(\text {chem) }}(t) / \Delta A_{s s}^{(\text {chem })}(-\ldots)$. $\Delta A_{\text {ss }}^{\text {(rot) }}$ and $\Delta A_{\text {ss }}^{\text {(chem) }}$ are the steady-state values of the rotational contribution and the chemical contribution, respectively, $E$ $=15.4 \times 10^{5} \mathrm{~V} \mathrm{~m}^{-1}, 20 \mu$ s pulse duration, $\lambda=565 \mathrm{~nm}$ 
particle-particle interactions may be considered negligible.

Electric dichroism may be caused by orientation of entire membrane fragments. Purple membrane fragments are oriented by longer lasting, exponentially decaying electric fields ${ }^{3,5}$ or in relatively low electric field strengths $\left(1 \times 10^{4} \mathrm{~V} \mathrm{~m}^{-1}\right)$ applied for sufficiently long time (1-2 $\min )^{7}$. The time constant for fragment orientation can be estimated using Perrin's equation ${ }^{18}$. Purple membranes may be considered as oblate discs with diameters between 0.5 and $1 \mu \mathrm{m}^{19}$; the rotational time constants for these dimensions are in the order of $100 \mathrm{~ms}$.

Although the longest relaxation time in the field-off process observed in this study is $\sim 100 \mathrm{~ms}$, it cannot be attributed to fragment orientation. Note that the rise up curve has already reached steady-state within $\sim 6 \mu$ s (see Figure 3). Now, according to the expressions developed by O'Konski et al. ${ }^{12}$, a steady-state obtained in $\sim 6 \mu$ s at a field strength of $15.4 \times 10^{5} \mathrm{~V} \mathrm{~m}^{-1}$ would require a permanent dipole moment of $1.3 \times 10^{-21} \mathrm{C} \mathrm{m}\left(3.9 \times 10^{8}\right.$ Debye) or an excess polarizability difference of 8.5 $\times 10^{-28} \mathrm{~F} \mathrm{~m}^{2}\left(7.6 \times 10^{-12} \mathrm{~cm}^{3}\right)_{2}$ As seen below, such unrealistically high values are not reached with bacteriorhodopsin. Therefore, fragment orientation can be excluded as the origin of the dichroism observed. In addition, pure fragment orientation is not expected to result in a chemical contribution to the absorbance changes.

A further possibility for orientational changes is the bending of membrane fragments. Because purple membranes are known to be rather rigid protein lipid lattices ${ }^{8}$, it is unlikely that the optical signals are caused by fragment bending.

We thus have to conclude that the bacteriorhodopsin molecules themselves are the source of the observed electric dichroism. The rigidity of the hexagonal lattice would, however, prevent the protein molecule from changing its overall position within the membrane $e^{20}$. It is more likely that only the parts of the protein involving the chromophore can move to a limited extent in the electric field; restricted chromophore motion is also apparent in the light-induced photocycle ${ }^{4,21-26}$. The irreversible absorbance change (bleaching) of old purple membrane fragments indicates that the electric field irreversibly affects the chromophore and possibly its immediate environment.

In summary, the electro-optic signals described in this study most probably results from electric field-induced structural changes which can be identified as limited orientational changes involving the chromophore.

\section{Orientation mechanism}

Applying the theory for restricted orientation to the field strength dependence of the reduced dichroism $\Delta A^{(r o t)} / A$, we obtain the mechanism for the angular displacement of the chromophore in bacteriorhodopsin. According to equations (3) and (9), the orientation factor $\Phi$ is proportional to $\Delta A^{(r o t)} / A$ and $\left|\beta^{2}+2 \gamma\right|$ is proportional to $E^{2}$, respectively. Therefore, the experimental data set $\Delta A^{(r o t)} / A$ can be fitted to the theoretical curves of $\Phi$ versus $\left|\beta^{2}+2 \gamma\right|$, see Figure 2 , by using proper scale factors ${ }^{27}$. It is found that, of the models considered, only case II of model $A$ gives satisfactory results; case I(A) and model B as well as free orientation can be excluded.

According to the observation of positive dichroism, we assume for simplicity that optical and electric axes are parallel $(\omega=0)$. In detail, Figure $9 a$ shows the comparison of $\Phi$ with $\Delta A^{(\text {rot })} / A$ (steady-state) for case II(A) with $\Delta \chi_{m}=0.35 \mathrm{rad}$ as a function of $\beta^{2}+2 \gamma$ for various ratios of $\beta^{2} / 2 \gamma ;(\gamma>0)$. The experimental data fit the curve for $\beta^{2} / 2 \gamma=0$ up to $E^{2}=5 \times 10^{11} \mathrm{~V}^{2} \mathrm{~m}^{-2}$, if at $E^{2}=1 \times 10^{11} \mathrm{~V}^{2} \mathrm{~m}^{-2}, \beta^{2}+2 \gamma=60$ and if $\Phi=0.19$ for $\Delta A^{(r o t)} / A=0.57$. The low field strength range is thus determined mainly by an induced dipole moment.

An additional slight increase in $\Phi$ becomer apparent at higher field strengths $\left(E^{2}>5 \times 10^{11} \mathrm{~V}^{2} \mathrm{~m}^{-2}\right)$ and can be attributed to a permanent dipole moment.

Figure $9 b$ summarizes the results. The entire experimental field strength range is quantitatively described by the maximum angular displacement $\Delta \chi_{m}=0.35 \mathrm{rad}$, the permanent dipole moment $p=4.7 \times 10^{-28} \mathrm{C} \mathrm{m}(140$ Debye) and the excess polarizability difference $\alpha_{1}-\alpha_{2}$ $=2.4 \times 10^{-30} \mathrm{~F} \mathrm{~m}^{2}\left(2.2 \times 10^{-14} \mathrm{C} \mathrm{m}^{3}\right)$.
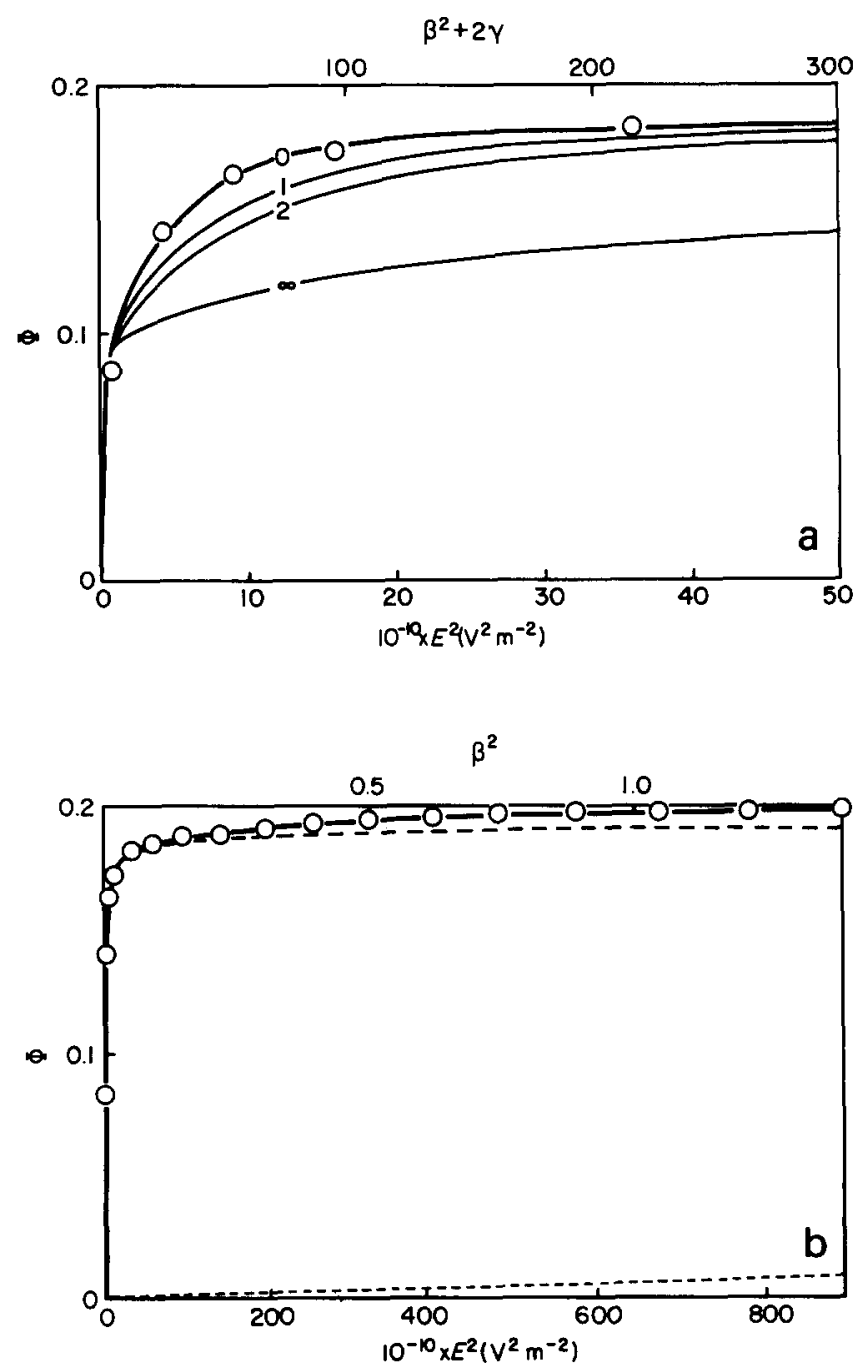

Figure 9 Electric field strength dependence of the orientation factor $\Phi$ in terms of $E^{2}, \beta^{2}+2 \gamma$ and $\beta^{2}$, for restricted angular displacement of the electric dipole axis from $\chi^{0}$ to $\chi^{0}-\Delta \chi_{m} ; \Delta \chi_{m}$ $=0.35 \mathrm{rad}\left(20^{\circ}\right)$. (a) Low field strength range for various ratios of $\beta^{2} / 2 \gamma(-)$. (b) Total experimental range of $E$ : --- , refers to the induced dipole contribution $(2 \gamma) ; \cdots$, refers to the permanent dipole contribution $\left(\beta^{2}\right)$ and - , covers both contributions. The comparison with the experimental data $(O)$ involves the assumption that the optical transition moment of the chromophore is parallel to the electric dipole axis $(\omega=0)$ 
Note that these numbers are only estimates for the apparent values of the electric parameters because no reliable correction factors for the internal and the directing fields ${ }^{28}$ can be given at present. In any case, the bacteriorhodopsin molecules in purple membranes represent a highly polarizable system. In conclusion, the field strength dependence of the electro-optical data suggest that the orientational change of the chromophore is restricted both in extent and in direction. The unidirectional angular displacement of the chromophore is towards the membrane normal and is limited to $\Delta \theta_{m}$ $\geqslant 0.35 \mathrm{rad}\left(20^{\circ}\right)$ corresponding to $\omega \geqslant 0$.

\section{Cooperativity}

The electric dipole moments of the orientation process, particularly the induced dipole moment, are comparatively large. The permanent dipole moment may originate in the $\alpha$-helices and in the paired polar groups (saltbridges) of bacteriorhodopsin. Since the seven $\alpha$-helical segments are almost parallel to the membrane normal ${ }^{8}$, the dipole moment of one $\alpha$-helix is not compensated by the oppositely directed moment of another $\alpha$-helix. Further, a segment contains, on a verage $30 \pm 3$ amino acid residues $^{29}$ and $\sim 70 \%$ is $\alpha$-helical ${ }^{30}$. Because the dipole moment of one hydrogen bonded $\mathrm{CO} \cdots \mathrm{HN}$ group in an $\alpha$-helix is $1.1 \times 10^{-29} \mathrm{C} \mathrm{m}(3.4 \text { Debye })^{31}$, the permanent dipole moment of these groups in one segment is $\sim 2.3$ $\times 10^{-28} \mathrm{C} \mathrm{m}\left(69\right.$ Debye). Since the estimate of $4.7 \times 10^{-28}$ $\mathrm{C} \mathrm{m}$ from the experimental data is larger, either ionic side groups of the residues appreciably contribute to the electric moment or the orienting unit comprises more than one bacteriorhodopsin molecule, or the directing field is much larger than the external field.

At $E \simeq 5 \times 10^{5} \mathrm{~V} \mathrm{~m}^{-1}$, a value up to which the field strength dependence of the dichroism suggests a constant polarizability value of $2.4 \times 10^{-30} \mathrm{~F} \mathrm{~m}^{2}$, the induced dipole moment is $1.2 \times 10^{-24} \mathrm{C} \mathrm{m}\left(3.6 \times 10^{5} \mathrm{Debye}\right)$. The chromophore orientation appears saturated at field strengths of $10-20 \times 10^{5} \mathrm{~V} \mathrm{~m}^{-1}$ whereas the induced dipole moment continues to increase and saturates at higher field intensities (unpublished data).

The primary sequence data ${ }^{29}$ and X-ray crystallographic results ${ }^{8}$ suggest that the positively charged side groups of lysine and arginine residues and the negatively charged carboxylate side chains of aspartic and glutamic acid residues could form ion pairs of the type $\mathrm{COO}^{-} \cdots \mathrm{HN}^{+}$. A maximum of 14 such pairs could be present per bacteriorhodopsin. The system $\mathrm{COOH}+\mathrm{N}=$ $\mathrm{COO}^{-}+\mathrm{HN}^{+}$is highly polarizable, yielding dipole moments up to $(3.8 \pm 0.5) \times 10^{-29} \mathrm{C} \mathrm{m}$ (11 Debye $)^{32}$. Thus the dipole moment arising from these groups might amount to $(5.3 \pm 0.7) \times 10^{-28} \mathrm{C} \mathrm{m}(160$ Debye). This value is very much smaller than the induced dipole moments at the field strengths applied. It is unlikely that this discrepancy is due to neglect of the internal field correction factor in the experimental value; it appears that the orienting unit is not one single bacteriorhodopsin molecule but represents a cluster of cooperatively coupled macromolecules; possibly the entire membrane fragment is involved. An induced dipole moment can also appear when the applied electric field increases the distance $l$ between oppositely charged groups: the dipole moment changes from $p_{0}$ to a value $p_{E}$ $=p_{0}+\Sigma e_{i} \Delta l_{i}$, where $e_{i}$ is the charge and $\Delta l_{i}$ is the fielddependent distance change of the ion pair $i$.
Evidence for cooperativity in purple membranes is frequently reported. For instance, cooperativity is indicated in the binding of retinal to apomembranes ${ }^{33}$, in the bleaching process ${ }^{34}$, as well as in the proton-pump cycle $^{4}$. It has been suggested that cooperative units larger than the trimer are involved ${ }^{33}$. If indeed the bacteriorhodopsin molecules orient the chromophores in a cooperative manner, then the electric axis of the orientation process is the vectorial sum of the various local contributions within the macromolecules. The local changes including the orientational displacement of the chromophore toward the membrane normal, are the conformational transitions induced by the electric field; leading to a finite value of:

$$
\Delta A^{(\text {chem })}=\sum_{i} \varepsilon_{i} \Delta c_{i}
$$

It is evident that the conformational changes must involve at least one structure with a different extinction coefficient.

\section{Reaction cycle}

The enormous difference between the time ranges of the rise up curve in the presence of the electric field and of the field-off response (see Figure 3) strongly suggests that the sequence of state changes caused in the electric field is different from the structural transitions occuring in the multistate field-off relaxation. The experimental data, therefore, suggest a cyclic reaction scheme which comprises at least five conformations in cooperative clusters of membrane-bound bacteriorhodopsin. The electric field causes a transition from state $b R_{1}$ to state $b R_{2}$, whereas the field-off relaxation reflects the passage through at least three, probably more, intermediate conformations.

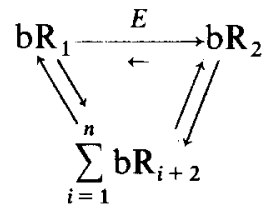

$(n \geqslant 3)$

In the light of the orientation mechanism discussed above, the step $b R_{1} \rightleftarrows b R_{2}$ may represent a 'synchronized', rapid alignment of ionic groups. These conformational changes appear to occur in the electric field in a concerted way and include a change in the chromophore transition moment from $1.2 \mathrm{rad}$ (state $b R_{1}$ ) to the angle $\leqslant 0.87 \mathrm{rad}\left(\right.$ state $b R_{2}$ ). We may now specify equation (30) in terms of these states by:

$$
\Delta A_{\sigma}=\left\{\Delta \varepsilon_{1, \sigma}(1-x)+\Delta \varepsilon_{2, \sigma} x\right\} c_{T}+\left(\bar{\varepsilon}_{2}-\bar{\varepsilon}_{1}\right) x c_{T}
$$

where $c_{T}=\left[b R_{1}\right]+\left[b R_{2}\right]$ is the total concentration of bacteriorhodopsin and $x=\left[\mathrm{bR}_{2}\right] / c_{r}$ is the fraction in state $\mathrm{bR}_{2}$.

Since the experimental data indicate restricted angular displacement of the chromophore to only one direction, $x$ $\leqslant 0.5$. At a sufficiently high electric field, where orientation is saturated, we have $x=0.5$. For this case, the extinction coefficient $\bar{\varepsilon}_{2}$ is readily calculated from $\Delta A^{\text {(chem) }}$ $=\left(\bar{\varepsilon}_{2}-\bar{\varepsilon}_{1}\right) x c_{T}$. For instance, at $565 \mathrm{~nm} \bar{\varepsilon}_{2}=54000 \mathrm{M}^{-1}$ $\mathrm{cm}^{-1}$ and at $400 \mathrm{~nm} \bar{\varepsilon}_{2}=21000 \mathrm{M}^{-1} \mathrm{~cm}^{-1}$

Although at the present accuracy of optical data recording the field-off relaxations appear to reflect at least 
four processes, the response curve is most probably a continuous spectrum of many local conformational transitions. Whereas the electric field acts simultaneously on all charged groups and dipoles of bacteriorhodopsin to cause a structural transition $\left(\mathrm{bR}_{1} \rightleftarrows \mathrm{bR} \mathrm{R}_{2}\right.$ ), the rearrangements in the absence of the field are more complex and face higher activation barriers, as is suggested by the larger relaxation times.

At present, it is not possible to connect the 'electrooptic cycle' represented in scheme (39) with details of the photocycle. However, some aspects of field and light effects are worth mentioning. It has been observed recently that in dry films of purple membranes there are spectral similarities between the field-induced intermediated and the first intermediate of the photocycle ${ }^{6}$.

The conformational changes supported by our electrooptic data clearly involve an orientational displacement of the chromophore toward the membrane normal. Interestingly, on the basis of Raman spectroscopic data it has been suggested that during the photocycle the chromophore rotates out of the membrane plane ${ }^{35}$. A further correlation between the photocycle and the electro-optic data is the appearance of cooperativity.

Although the cyclic nature and the kinetics of the electric field induced structural changes suggest common features with the photocycle, further studies are in progress in order to identify the effect of electric fields on the dynamic properties of bacteriorhodopsin. It should, however, be mentioned that the data on bacteriorhodopsin are suggestive of a possibly general mechanism for the interaction of membrane electric fields with transport and gating proteins, in particular those of excitable biomembranes. The field dependence of the ion permeability changes underlying the nerve impulse and other membrane potential changes would then be based on a polarization mechanism where the distances between charged groups of the ion flow gating proteins depend on the intensity of the membrane electric field.

\section{Acknowledgement}

We thank Professor K. Rosenheck and A. Zakaria of the Weizmann Institute of Science for the supply of purple membrane samples. The technical help of C. R. Rabl, D. Schallreuter and J. Rohner is gratefully acknowledged.

\section{References}

1 Oesterhelt, D. and Stoeckenius, W. Nature (London) New Biol. $1971,233,149$

2 Oesterhelt, D. and Stoeckenius, W. Proc. Natl. Acad. Sci. USA 1973, 70, 2853

3 Shinar, R., Druckmann, S., Ottolenghi, M. and Korenstein, R. Biophys. J. 1977, 19, 1

4 Hess, B., Korenstein, R. and Kuschmitz, D. in 'Energetics and Structure of Halophilic Microorganisms' (Eds. S. R. Caplan and M. Ginzburg), Elsevier/North-Holland Biochemical Press, Amsterdam, 1978, p. 89

5 Tsuji, K. and Rosenheck, K. in 'Electro-Optics and Dielectrics of Macromolecules and Colloids' (Ed. B. R. Jennings), Plenum Press, New York, 1979, p. 77

6 Borisevitch, G. P., Lukashev, E. P., Kononenko, A. A. and Rubin, A. B. Biochim. Biophys. Acta 1979, 546, 171

7 Eisenbach, M., Weissmann, C., Tanny, G. and Caplan, S. R. FEBS Lett. 1977, 81, 77

Henderson, R. J. Mol. Biol. 1975, 93, 123

9 Kriebel, A. N. and Albrecht, A. C. J. Chem. Phys. 1976, 65, 4575

10 Ebrey, T. G., Becher, B., Mao, B., Kilbride, P. and Honig, B. $J$. Mol. Biol. 1977, 112, 377
11 Heyn, M. P., Cherry, R. J. and Müller, U. J. Mol. Biol. 1977, 117 , 607

12 O'Konski, C. T., Yoshioka, K. and Orttung, W. H. J. Phys, Chem. $1959,63,1558$

13 Fredericq, E. and Houssier, C. 'Electric Dichroism and Electric Birefringence', Clarendon Press, Oxford 1973

14 Oesterhelt, D. and Hess, B. Eur. J. Biochem. 1973, 37, 316

15 Schallreuter, D. and Neumann, E. Ber. Bunsenges. Phys. Chem. in press

16 Rigler, R., Rabl, C. R. and Jovin, T. M. Rev. Sci. Instrum. 1974, 45, 580

17 Neumann, E. in 'Electro-Optics and Dielectrics of Macromolecules and Colloids' (Ed. B. R. Jennings), Plenum Press, New York, 1979, p. 233

18 Perrin, F. J. Phys. Radium. 1926, 7, 390

19 Blaurock, A. E. and Stoeckenius, W. Nature (London) New Biol. 1971, 233, 152

20 Razi Naqui, K., Gonzalez-Rodriguez, J., Cherry, R. J. and Chapman, D. Nature (London) New Biol. 1973, 245, 249

21 Konishi, T. and Packer, L. FEBS Lett. 1978, 92, 1

22 Lewis, A., Marcus, M. A., Ehrenberg, B. and Crespi, H. Proc. Natl. Acad. Sci. USA 1978, 75, 4642

23 Gillbro, T. Biochim. Biophys. Acta 1978, 504, 175

24 Lozier, R. H. and Niederberger, W. Fed. Proc. 1977, 36, 1805

25 Schulten, K. and Tavan, P. Nature (London) 1978, 272, 85

26 Hurley, J. B., Becher, B. and Ebrey, T. G. Nature (London) 1978 , 272, 87

27 Yoshioka, K. and Watanabe, H. in 'Physical Principles and Techniques of Protein Chemistry, Part A' (Ed. S. J. Leach), Academic Press Inc., New York, 1969, p. 339

28 Böttcher, C. J. F. in 'Theory of Electric Polarization', Elsevier Sci. Pub. Co., Amsterdam, 1973

29 Ovchinnikov, Yu. A., Abdulaev, N. G., Feigina, M. Yu., Kiselev, A. V. and Lobanov, N. A. FEBS Lett. 1979, 100, 219

30 Becher, B. and Cassim, J. Y. Biophys. J. 1976, 16, 1183

31 Wada, A. in 'Poly- $\alpha$-Amino Acids' (Ed. G. Fasman), Marcel Dekker, New York, 1967, 369

32 Zundel, G. in 'The Hydrogen Bond, Recent Developments in Theory and Experiments' (Eds. P. Schuster, G. Zundel and C. Sandorfy), North-Holland Publ. Co., Amsterdam, 1976 Rehorek, M. and Heyn, M. P. Biochemistry 1979, 18, 4977 Becher, B. and Cassim, J. Y. Biophys. J. 1977, 19, 285 Lewis, A. Phil Trans. R. Soc. London (A) 1979, 293, 315

\section{Nomenclature}

Greek letters

$\alpha_{1}, \alpha_{2} \quad$ Electric polarizabilities along the symmetry axis and the transverse axis, respectively $\left(\mathrm{F} \mathrm{m}^{2}\right)$ $\beta \quad$ Permanent dipole term

$\gamma \quad$ Induced dipole term

$\bar{\varepsilon}_{i} \quad$ Molar absorption coefficient of randomly distributed species $i\left(\mathrm{M}^{-1} \mathrm{~cm}^{-1}\right)$

$\varepsilon_{i, \sigma}^{E} \quad$ Molar absorption coefficient of species $i$ for the light polarization mode $\sigma$ in the electric field Angle between the chromophore transition moment and the electric field direction (rad)

$\kappa \quad$ Extinction index

$\lambda \quad$ Wavelength $(\mathrm{nm})$

$\mu \quad$ Optical transition dipole moment $(\mathrm{C} \mathrm{m}$ )

$\sigma \quad$ Light polarization angle relative to the electric field direction (rad)

$\tau \quad$ Relaxation time (s)

$\Phi \quad$ Orientation factor

$\varphi \quad$ Azimuthal angle relative to the electric field direction (rad)

$\psi \quad$ Angle between the chromophore transition moment and the membrane normal (rad)

$\omega$ Angle between the chromophore transition moment and the electrical symmetry axis of the orienting unit (rad)

$\chi \quad$ Angle between the electrical symmetry axis of the orienting unit and the electric field (rad) 
Roman letters

$A_{\lambda} \quad$ Absorbance per cm at wavelength $\lambda$

$A_{\lambda, \sigma}^{E} \quad$ Absorbance per $\mathrm{cm}$ in the presence of the electric field at the wavelength $\lambda$ and the polarization direction $\sigma$ to the electric field

$\Delta A_{\sigma} \quad$ Absorbance change due to the electric field

$\Delta A_{\sigma} / A \quad$ Reduced absorbance change

$\Delta A^{\text {(chem) }}$ Chemical contribution to the absorbance change

$\Delta A_{\sigma}^{(r o t)} \quad$ Rotational contribution to the absorbance change

$c_{i} \quad$ Concentration of species $i(M)$

$E \quad$ Electric field strength $\left(\mathrm{V} \mathrm{m}^{-1}\right)$

$I \quad$ Intensity of transmitted light (V)

$\Delta I \quad$ Change of $I$ caused by the electric field (V)

$k \quad$ Boltzmann constant $\left(\mathrm{J} \mathrm{K}^{-1}\right)$

$p \quad$ Electric permanent dipole moment $(\mathrm{C} \mathrm{m})$

$t \quad$ Time (s)

$T \quad$ Absolute temperature (K)
$U \quad$ Total potential energy of the orienting unit in the electric field (J)

$U_{0} \quad$ Interaction energy of the orienting unit with the electric field due to permanent and induced dipole moments $(\mathrm{J})$

$U_{r} \quad$ Restriction energy $(\mathrm{J})$

\section{Superscripts}

The superscripts 0 and $E$ used for $\theta, \psi, \chi$ etc. refer to the values in the absence and the presence of the electric field, respectively.

\section{Subscripts}

The subscripts $\sigma, \|$ and $\perp$ for $\varepsilon$ and $A$ refer to the values along the direction $\sigma$ rad, parallel and perpendicular to the electric field, respectively.

The subscripts $s$ and $s s$ for $A$ refer to the values in the saturated ranges and the steady state, respectively. 